\title{
STRONG LAWS OF LARGE NUMBERS FOR ARRAYS OF ROWWISE CONDITIONALLY INDEPENDENT RANDOM ELEMENTS ${ }^{1}$
}

\author{
RONALD FRANK PATTERSON \\ Georgia State University \\ Department of Mathematics and Computer Science \\ Atlanta, GA 30303 U.S.A. \\ ABOLGHASSEM BOZORGNIA \\ Mashhad University \\ Department of Statistics \\ Mashhad, IRAN \\ ROBERT LEE TAYLOR \\ University of Georgia \\ Department of Statistics \\ Athens, GA 30602 U.S.A.
}

\begin{abstract}
Let $\left\{X_{n k}\right\}$ be an array of rowwise conditionally independent random elements in a separable Banach space of type $p, 1 \leq p \leq 2$.

Complete convergence of $n^{-1 / r} \sum_{k=1}^{n} X_{n k}$ to $0,0<r<p \leq 2$ is obtained by using various conditions on the moments and conditional means. A Chung type strong law of large numbers is also obtained under suitable moment conditions on the conditional means.
\end{abstract}

Key words: Strong law of large numbers, type $p$, rowwise conditionally independent, complete convergence.

AMS (MOS) subject classifications: $\quad 60 \mathrm{~B} 12$.

\section{INTRODUCTION AND PRELIMINARIES}

Let $(8,\|\cdot\|)$ be a real separable Banach space. Let $(\Omega, \mathcal{A}, P)$ denote a probability space. A random element (r.e.) $X$ in $\&$ is a function from $\Omega$ into $\&$ which is $\mathcal{A}$-measurable with respect to the Borel subsets $B(8)$. The $r$ th absolute moment of a random element $X$ is $E\|X\|^{r}$ where $E$ is the expected value of the random variable $\|X\|^{r}$. The expected value of

\footnotetext{
${ }^{1}$ Received: August, 1992. Revised: January, 1993.
} 
a random element $X$ is defined to be the Bochner integral (when $E\|X\|<\infty$ ) and is denoted by $E X$. The concepts of independence and identical distributions for real-valued random variables extend directly to 8 . A separable Banach space is said to be of (Rademacher) type $p, 1 \leq p \leq 2$, if there exist a constant $C$ such that

$$
E\left\|\sum_{k=1}^{n} X_{k}\right\|^{p} \leq C \sum_{k=1}^{n} E\left\|X_{k}\right\|^{p}
$$

for all independent random elements $X_{1}, \ldots, X_{n}$ with zero means and finite $p$ th moments. The sequence of random elements $\left\{X_{n}\right\}$ is said to be conditionally independent if there exists a sub$\sigma$-field $\zeta$ of $\mathcal{A}$ such that for each positive integer $m$

$$
P\left[\bigcap_{i=1}^{m}\left[X_{i} \in B_{i}\right] \mid \zeta\right]=\prod_{i=1}^{m} P\left[X_{i} \in B_{i} \mid \zeta\right] a . s .
$$

where $P\left[X_{i} \in B_{i} \mid \zeta\right]$ denotes the conditional probability of the random element $X_{i}$ being in the Borel set $B_{i}$ given the sub- $\sigma$-field $\zeta$. Independent random elements are conditionally independent with respect to the trivial $\sigma$-field $\{\emptyset, \mathcal{A}\}$.

Throughout this paper $\left\{X_{n k}: 1 \leq k \leq n, n \geq 1\right\}$ will denote rowwise conditionally independent random elements in $\&$ such that $E X_{n k}=0$ for all $n$ and $k$. The first major result of this paper shows that

$$
\frac{1}{n^{1 / n}} \sum_{k=1}^{n} X_{n k} \rightarrow 0 \text { completely }
$$

where complete convergence is defined (as in Hsu and Robbins [5]) by

$$
\sum_{n=1}^{\infty} P\left[\left\|\frac{1}{n^{1 / r}} \sum_{k=1}^{n} X_{n k}\right\|>\epsilon\right]<\infty
$$

for each $\epsilon>0$. The second major result is a Chung type strong law of large numbers (SLLN) which provides

$$
\frac{1}{a_{n}} \sum_{k=1}^{n} X_{n k} \rightarrow 0 \text { a.s. }
$$

where $a_{n}<a_{n+1}$ and $\lim _{n \rightarrow \infty} a_{n}=\infty$. For comparisons with (1.2) and (1.4), a brief partial review of previous results will follow.

Erdös [4] showed that for an array of i.i.d. random variables $\left\{X_{n k}\right\},(1.2)$ holds if and only if $E\left|X_{11}\right|^{2 r}<\infty$. Jain [8] obtained a uniform SLLN for sequences of i.i.d. r.e.'s in a separable Banach space of type 2 which would yield (1.2) with $r=1$ for an array of r.e.'s $\left\{X_{n k}\right\}$. Woyczynski [12] showed that

$$
\frac{1}{n^{1 / r}} \sum_{k=1}^{n} X_{k} \rightarrow 0 \text { completely }
$$


for any sequence $\left\{X_{n}\right\}$ of independent r.e.'s in a Banach space of type $p, 1 \leq r<p \leq 2$ with $E X_{n}=0$ for all $n$ which is uniformly bounded by a random variable $X$ satisfying $E|X|^{r}<\infty$. Recall that an array $\left\{X_{n k}\right\}$ of r.e.'s is said to be uniformly bounded by a random variable $X$ if for all $n$ and $k$ and for every real number $t>0$

$$
P\left[\left\|X_{n k}\right\|>t\right] \leq P[|X|>t] .
$$

Hu, Moricz and Taylor [7] showed that Erdös' result could be obtained by replacing the i.i.d. condition by the uniformly bounded condition (1.6). Taylor and $\mathrm{Hu}$ [9] obtained complete convergence in type $p$ spaces, $1<p \leq 2$ for uniformly bounded, rowwise independent r.e.'s. Bozorgnia, Patterson and Taylor [1] obtained a more general result by replacing the assumption of uniformly bounded random elements with moment conditions. One complete convergence result of this paper, given in Section 2, is obtained by assuming a condition on the conditional means and extends the result in Bozorgnia et. al [1].

If $\left\{X_{n}\right\}$ is a sequence of independent (but not necessarily identically distributed) r.v.'s, Chung's SLLN yield (1.4) for r.v.'s if $\Psi(t)$ is a positive, even, continuous function such that either

$$
\Psi(t) \downarrow \text { as }|t| \uparrow \infty
$$

and

$$
\sum_{n=1}^{\infty} \frac{E \Psi\left(X_{n}\right)}{\Psi\left(a_{n}\right)}<\infty
$$

where $\left\{a_{n}\right\}$ is a sequence of real numbers such that $a_{n}<a_{n+1}$ and $\lim _{n \rightarrow \infty} a_{n}=\infty$ hold, or

$$
\frac{\Psi(t)}{|t|} \uparrow \text { and } \frac{\Psi(t)}{|t|^{2}} \downarrow \text { as }|t| \uparrow \infty
$$

$E X_{n}=0$ and (1.8) holds where $\uparrow$ and $\downarrow$ denote monotone increasing and monotone decreasing respectively.

$\mathrm{Wu}$, Taylor and $\mathrm{Hu}[6]$ considered SLLN's for arrays of rowwise independent random variables, $\left\{X_{n i}: 1 \leq i \leq n, n \geq 1\right\}$. They obtained Chung type SLLN's under the more general conditions:

$$
\frac{\Psi(|t|)}{|t|^{r}} \uparrow \text { and } \frac{\Psi(|t|)}{|t|^{r+1} \downarrow} \text { as }|t| \uparrow
$$

where $\Psi(t)$ is a positive, even function and $r$ is a nonnegative integer,

$$
\begin{gathered}
E X_{n i}=0 \\
\sum_{n=1}^{\infty} \sum_{i=1}^{n} \frac{E\left(\Psi\left(X_{n i}\right)\right)}{\Psi\left(a_{n}\right)}<\infty
\end{gathered}
$$


and

$$
\sum_{n=1}^{\infty}\left[\sum_{i=1}^{n} E\left(\frac{X_{n i}}{a_{n}}\right)^{2}\right]^{2 k}<\infty
$$

where $k$ is a positive integer and $\left\{a_{n}\right\}$ is a sequence of positive real numbers defined in (1.4). Combinations of Conditions (1.10), (1.11), (1.12) and (1.13) for different values of $r$ will imply that

$$
\frac{1}{a_{n}} \sum_{i=1}^{n} X_{n i} \rightarrow 0 \text { a.s. }
$$

Bozorgnia, Patterson and Taylor [2] obtained Banach space versions of $\mathrm{Hu}$, Taylor and Wu's results using the modified conditions:

$$
\frac{\Psi(|t|)}{|t|^{r}} \uparrow \text { and } \frac{\Psi(|t|)}{|t|^{r+p-1} \downarrow} \text { as }|t| \uparrow
$$

for some nonnegative integer $r$, where the separable Banach space is of type $p, 1 \leq p \leq 2$,

$$
\begin{gathered}
E X_{n i}=0 \\
\sum_{n=1}^{\infty} \sum_{i=1}^{n} \frac{E\left(\Psi\left(\left\|X_{n i}\right\|\right)\right)}{\Psi\left(a_{n}\right)}<\infty
\end{gathered}
$$

and

$$
\sum_{n=1}^{\infty}\left[\sum_{i=1}^{n} E\left(\left\|\frac{X_{n i}}{a_{n}}\right\|^{p}\right)\right]^{p k}<\infty
$$

where $k$ is a positive integer.

In Section 2 of this paper, SLLN's for arrays of rowwise conditionally independent r.e.'s are obtained for Banach spaces under conditions similar to those of Chung [3], Hu, Taylor and $\mathrm{Wu}$ [6] and Bozorgnia, Patterson and Taylor [2] with appropriate conditions on the conditional means. These new results address the question of possible exchangeability extensions in the affirmative, and in addition, provide a class of new results for conditionally independent random elements. A generic constant, $C$, will be used throughout the paper.

\section{MAIN RESULTS}

A lemma by Wozczynski [12], is crucially used in the proofs of the major results, Theorems 2.2 and 2.3 , and is stated here for future reference.

Lemma 2.1: $\quad$ Let $1 \leq p \leq 2$ and $q \geq 1$. The following properties are equivalent:

(i) The separable Banach space, 8 , is of type $p$.

(ii) There exist a constant $C$ such that for all independent r.e.'s $X_{1}, \ldots, X_{n}$ in $\&$ with 


$$
\begin{aligned}
& E X_{i}=0, \text { and } E\left\|X_{i}\right\|^{q}<\infty, i=1,2, \ldots, n \\
& \qquad E\left\|\sum_{i=1}^{n} X_{i}\right\|^{q} \leq C E\left(\sum_{i=1}^{n}\left\|X_{i}\right\|^{p}\right)^{q / p} .
\end{aligned}
$$

The constant $C$ depends only on the Banach space 8 and not on $n$. Moreover, throughout this section $C$ will denote a generic constant which is not necessarily the same each time used but is always independent of $n$.

Theorem 2.2: Let $\left\{X_{n k}\right\}$ be an array of rowwise conditionally independent random elements in a separable Banach space of type $p, 1 \leq p \leq 2$. If

and

(i) $\underset{1 \leq k \leq n}{\sup } E\left\|X_{n k}\right\|^{\nu}=O\left(n^{\alpha}\right), \alpha \geq 0$ where $\nu\left(\frac{1}{r}-\frac{1}{p}\right)>\alpha+1,0<r<p \leq 2$

(ii) for all $\eta>0$,

$$
\sum_{n=1}^{\infty} P\left(\left\|\frac{1}{n^{1 / r}} \sum_{k=1}^{n} E_{\zeta} X_{n k}\right\|>\eta\right)<\infty
$$

where $E_{\zeta}$ is the conditional expectation with respect to an appropriate $\sigma$-field that gives conditional independence, then

$$
\frac{1}{n^{1 / r}} \sum_{k=1}^{n} X_{n k} \rightarrow 0 \text { completely. }
$$

Proof: Let $\epsilon>0$ be given. By Markov's inequality,

$$
\begin{aligned}
\sum_{n=1}^{\infty} P\left(\left\|\frac{1}{n^{1 / r}} \sum_{k=1}^{n} X_{n k}\right\|>\epsilon\right) \leq \sum_{n=1}^{\infty} P\left(\left\|\frac{1}{n^{1 / r}} \sum_{k=1}^{n}\left(X_{n k}-E_{\zeta} X_{n k}\right)\right\|>\frac{\epsilon}{2}\right) \\
+\sum_{n=1}^{\infty} P\left(\left\|\frac{1}{n^{1 / r}} \sum_{k=1}^{n} E_{\zeta} X_{n k}\right\|>\frac{\epsilon}{2}\right) \\
\leq C \sum_{n=1}^{\infty} E E_{\zeta}\left\|\frac{1}{n^{1 / r}} \sum_{k=1}^{n}\left(X_{n k}-E_{\zeta} X_{n k}\right)\right\|^{\nu} \\
+\sum_{n=1}^{\infty} P\left(\left\|\frac{1}{n^{1 / r}} \sum_{k=1}^{n} E_{\zeta} X_{n k}\right\|>\frac{\epsilon}{2}\right) .
\end{aligned}
$$

By Lemma 2.1 and Hölder's inequality, the first term in (2.2) is bounded by

$$
\begin{gathered}
C \sum_{n=1}^{\infty} E\left(E_{\zeta}\left\|\frac{1}{n^{1 / r}} \sum_{k=1}^{n}\left(X_{n k}-E_{\zeta} X_{n k}\right)\right\|^{\nu}\right) \leq C \sum_{n=1}^{\infty} \frac{n^{\nu / p-1}}{n^{\nu / r}} E\left(\sum_{k=1}^{n}\left\|X_{n k}-E_{\zeta} X_{n k}\right\|^{\nu}\right) \\
\leq C \sum_{n=1}^{\infty} \frac{n^{\nu / p-1}}{n^{\nu / r}} \cdot 2^{\nu} \sum_{k=1}^{n} E\left\|X_{n k}\right\|^{\nu} \\
\leq C \sum_{n=1}^{\infty} \frac{n^{\nu / p} \cdot n^{\alpha}}{n^{\nu / r}}
\end{gathered}
$$




$$
=C \sum^{\infty} n^{-\nu\left(\frac{1}{r}-\frac{1}{p}\right)+\alpha}<\infty .
$$

The second term of $(2.1)$ is finite by $(i i)=1$ Thus, the result follows.

Remark 1: Condition (ii) can be replaced by the condition $E\left\|E_{\zeta} X_{n 1}\right\|^{p}=O\left(n^{-\beta}\right)$, $\beta>\frac{2 r-p}{r}$, if the r.e.'s are conditionally i.i.d. or rowwise infinitely exchangeable. If $0<r<1$, and $p / r>2$, then $\beta$ can be nonpositive and the bound for each row can increase.

Remark 2: If the random elements are independent with zero means, then condition (ii) is identically zero when $\zeta$ is chosen to be the trivial $\sigma$-field, $\{\emptyset, \mathcal{A}\}$. Thus, Theorem 2.2 generalizes the results of Bozorgnia et. al [1].

Remark 3: Condition (i) implies condition (6.2.2) in Theorem 6.2.3 of Taylor, Daffer and Patterson [10]. Condition (6.2.2) was given as:

$$
\sum_{n=1}^{\infty} \frac{E\left(\left\|X_{n 1}\right\|^{p q}\right)}{n^{q(p-1)}}<\infty .
$$

Letting $\nu=p q$ and $r=1$, it follows that the third inequality in the proof of Theorem 2.2 is majorized by

$$
\begin{gathered}
C \sum_{n=1}^{\infty} \frac{n \cdot n^{\nu / p-1}}{n^{\nu / r}} \cdot \sup _{1 \leq k \leq n} E\left\|X_{n k}\right\|^{\nu}=C \sum_{n=1}^{\infty} \frac{n^{\nu / p}}{n^{\nu}} \cdot E\left\|X_{n 1}\right\|^{\nu} \\
\leq C \sum_{n=1}^{\infty} \frac{n^{q} \cdot n^{\alpha}}{n^{p q}} \\
=C \sum_{n=1}^{\infty} n^{-q(p-1)+\alpha}
\end{gathered}
$$

which is a substantial improvement of Theorem 6.2.3 of Taylor et. al [10]. Moreover, Condition (6.2.1) of Theorem 6.2.3 in Taylor et. al [10] implies Condition (ii) of Theorem 2.2 since for $\left\{X_{n k}\right\}$ rowwise infinitely exchangeable and $r=1$,

$$
\begin{gathered}
\sum_{n=1}^{\infty} P\left[\left\|\frac{1}{n} \sum_{k=1}^{n} E_{\zeta} X_{n k}\right\|>\eta^{1 / \nu}\right] \leq \sum_{n=1}^{\infty} P\left(\left\|E_{\zeta} X_{n 1}\right\|^{\nu}>\eta\right) \\
=\sum_{n=1}^{\infty} \int P_{\zeta}\left(\left\|E_{\zeta} X_{n 1}\right\|^{\nu}>\eta\right) d \mu_{n}\left(P_{\zeta}\right) \\
=\sum_{n=1}^{\infty} \mu_{n}\left\{P_{\zeta}:\left\|E_{\zeta} X_{n 1}\right\|^{\nu}>\eta\right\}<\infty
\end{gathered}
$$

where $\mu_{n}$ denotes the mixing measure for the exchangeable sequence $\left\{X_{n 1}, X_{n 2}, \ldots\right\}$ and $P_{\zeta}$ denotes the conditional probability.

The next result is a Chung type SLLN for arrays of rowwise conditionally independent r.e.'s in a separable Banach space of type $p, 1 \leq p \leq 2$. Let $\left\{a_{n}\right\}$ be a sequence of positive real 
numbers such that $a_{n}<a_{n+1}$ and $\lim _{n \rightarrow \infty} a_{n}=\infty$. Let $\Psi(t)$ be the positive, even function defined in (1.15).

Theorem 2.3: Let $\left\{X_{n i}\right\}$ be an array of rowwise, conditionally independent random elements in a separable Banach space of type $p, 1 \leq p \leq 2$ such that $E X_{n i}=0$ for all $n$ and $i$. Let $\Psi(t)$ satisfy (1.15) for some $r \geq 2$. If $\left\{a_{n}\right\}$ is a sequence of positive real numbers such that $a_{n}<a_{n+1}$ and $\lim _{n \rightarrow \infty} a_{n}=\infty$ and if

$$
\frac{1}{a_{n}} \sum_{i=1}^{n} E_{\zeta} X_{n i} \rightarrow 0 \text { completely }
$$

and if for some positive integer $k$

$$
\sum_{n=1}^{\infty} E\left(\sum_{i=1}^{n} E_{\zeta}\left\|\frac{X_{n i}}{a_{n}}\right\|^{p}\right)^{p k}<\infty
$$

then Condition (1.17) implies that

$$
\frac{1}{a_{n}} \sum_{i=1}^{n} X_{n i} \rightarrow 0 \text { a.s. }
$$

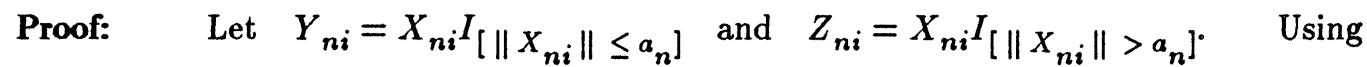
Markov's inequality and Condition (1.1.7), it follows that the two sequences

$$
\left\{\sum_{i=1}^{n}\left(\frac{X_{n i}}{a_{n}}\right)\right\} \text { and }\left\{\sum_{i=1}^{n}\left(\frac{Y_{n i}}{a_{n}}\right)\right\}
$$

are equivalent. Conditions (1.15), (1.16) and (1.17) imply that

$$
\begin{gathered}
\sum_{n=1}^{\infty} \sum_{i=1}^{n}\left\|E\left(\frac{Y_{n i}}{a_{n}}\right)\right\|=\sum_{n=1}^{\infty} \sum_{i=1}^{n}\left\|E\left(\frac{Z_{n i}}{a_{n}}\right)\right\| \\
\leq \sum_{n=1}^{\infty} \sum_{i=1}^{n} \frac{E\left(\Psi\left(\left\|X_{n i}\right\|\right)\right)}{\Psi\left(a_{n}\right)}<\infty .
\end{gathered}
$$

Next,

$$
\begin{gathered}
\left\|\frac{1}{a_{n}} \sum_{i=1}^{n}\left(Y_{n i}-E Y_{n i}\right)\right\| \leq\left\|\frac{1}{a_{n}} \sum_{i=1}^{n}\left(Y_{n i}-E_{\zeta} Y_{n i}\right)\right\| \\
+\left\|\frac{1}{a_{n}} \sum_{i=1}^{n} E_{\zeta} Y_{n i}\right\|+\left\|\frac{1}{a_{n}} \sum_{i=1}^{n} E Y_{n i}\right\| .
\end{gathered}
$$

The last term of (2.6) converges to 0 by (2.5). By Condition (2.3)

if and only if

$$
\left\|\frac{1}{a_{n}} \sum_{i=1}^{n} E_{\zeta} Y_{n i}\right\| \rightarrow 0 \text { completely }
$$

$$
\left\|\frac{1}{a_{n}} \sum_{i=1}^{n} E_{\zeta} Z_{n i}\right\| \rightarrow 0 \text { completely. }
$$

But, (2.7) follows from (1.17) since 


$$
\begin{gathered}
E\left\|\frac{1}{a_{n}} \sum_{i=1}^{n} E_{\zeta} Z_{n i}\right\| \leq E\left(E_{\zeta}\left\|\sum_{i=1}^{n} \frac{Z_{n i}}{a_{n}}\right\|\right) \\
\leq \sum_{i=1}^{n} E\left\|\frac{Z_{n i}}{a_{n}}\right\| \\
\leq \sum_{i=1}^{n} \frac{E\left(\Psi\left(\left\|Z_{n i}\right\|\right)\right)}{\Psi\left(a_{n}\right)} \\
\leq \sum_{i=1}^{n} \frac{E\left(\Psi\left(\left\|X_{n i}\right\|\right)\right)}{\Psi\left(a_{n}\right)} .
\end{gathered}
$$

Thus, it suffices to show that

$$
\sum_{i=1}^{n} \frac{Y_{n i}-E_{\zeta} Y_{n i}}{a_{n}} \rightarrow 0 \text { a.s. }
$$

Let $W_{n i}=\frac{Y_{n i}}{a_{n}}-\frac{E_{\zeta} Y_{n i}}{a_{n}}$ for all $n$ and $i$. Then, $\left\|W_{n i}\right\| \leq 2$ and $E_{\zeta} W_{n i}=0$. Now by Lemma 2.1,

$$
\begin{gathered}
E\left\|\sum_{i=1}^{n} W_{n i}\right\|^{p k(r+1)}=E\left(E_{\zeta}\left\|\sum_{i=1}^{n} W_{n i}\right\|^{p k(r+1)}\right) \\
\leq C E\left(E_{\zeta}\left(\sum_{i=1}^{n}\left\|W_{n i}\right\|^{p}\right)^{k(r+1)}\right) \\
=C E \sum^{*}\left(\begin{array}{c}
k(r+1) \\
s_{1}, \ldots, s_{n}
\end{array}\right) E_{\zeta}\left\|W_{n 1}\right\|^{p s_{1} \ldots E_{\zeta}\left\|W_{n n}\right\|^{p s_{n}}}
\end{gathered}
$$

where the sum $\sum^{*}$ is over all choices of nonnegative integers $s_{1}, \ldots, s_{n}$ such that $\sum_{i=1}^{n}$ $s_{i}=k(r+1)$. Now (2.9) can be shown to be summable with respect to $n$ following the same steps as in the proof of Theorem 2.2 of Bozorngia et. al [2] for the case $s_{i} p \geq r+1$ for at least one $s_{i}$. The case $s_{i} p<r+1$ for all $i$ is accomplished by using (2.4) instead of (1.18). Hence, the result follows.

Remark 4: Theorem 2.2 extends the random variable result in $\mathrm{Hu}$, Taylor and $\mathrm{Wu}[6]$ for $p=2$ and the random element results in Bozorgnia, Patterson and Taylor [2] to the class of conditionally independent random variables and random elements. Again, if the r.e.'s are rowwise independent with zero means, then Condition (2.3) is equal to zero via the trivial $\sigma$ field, and (2.4) becomes (1.18) with the trivial $\sigma$-field.

\section{ACKNOWLEDGEMENTS}

The research by the first author was supported in part by the National Science Foundation under grant \#DMS 8914503. The research for the first and second authors was 
mainly completed while at the Department of Statistics, University of Georgia, Athens, GA.

\section{REFERENCES}

[1] Bozorgnia, A., Patterson, R.F., and Taylor, R.L., On strong laws of large numbers for arrays of rowwise independent random elements, Internat. J. Math. \& Math. Sci., (to appear).

[2] Bozorgnia, A., Patterson, R.F., and Taylor, R.L., Chung type strong laws for arrays of random elements and bootstrapping, Statistics Technical Report 92-8, University of Georgia, (1992).

[3] Chung, K.L., Note on some strong laws of large numbers, Amer. J. Math 69, 189-192, (1947).

[4] Erdös, P., On a theorem of Hsu and Robbins, Ann. Math. Statistics 20, 286-291, (1949).

[5] Hsu, P.L. and Robbins, H., Complete convergence and the law of large numbers, Proc. Nat. Sci. USA, 33, 25-31, (1947).

[6] $\mathrm{Hu}$, T.C., Taylor, R.L. and Wu, J.S., On the strong law for arrays and for the bootstrap mean and variance, Statistics Technical Report 91-16, University of Georgia, (1991).

[7] Hu, T.C., Moricz, F. and Taylor, R.L., Strong law of large numbers for arrays of rowwise independent random variables, Actua Math. Hung. 54, 153-162, (1986).

[8] Jain, N.C., Tail probabilities for sums of independent Banach space random variables, Z. Wahr. V. Geb. 33, 155-166, (1975).

[9] Taylor, R.L., and Hu, T.C., Strong laws of large numbers for arrays of rowwise independent random elements, Internat. J. Math E Math. Sci. 4, 805-814, (1987).

[10] Taylor, R.L., Daffer, P. and Patterson, R.F., Limit Theorems for Sums of Exchangeable Random Variables, Rowman \& Allanheld Publishers, Totowa N.J. 1985.

[11] Woyczynski, W.A., Geometry and martingales in Banach spaces, Part II, Advances in Probability 4, Dekker, 267-517, (1978).

[12] Woyczynski, W.A., On Marcinkiewicz-Zygmund laws of large numbers, Prob. and Math. Stat. 1, 117-131, (1980). 


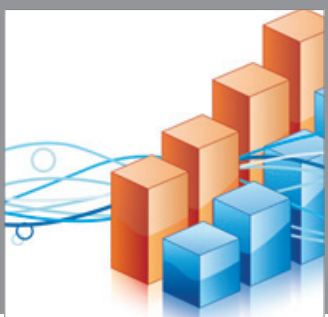

Advances in

Operations Research

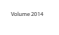

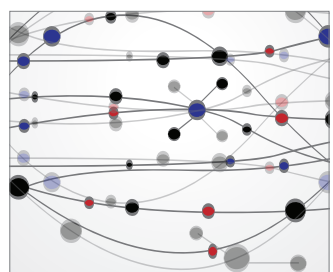

\section{The Scientific} World Journal
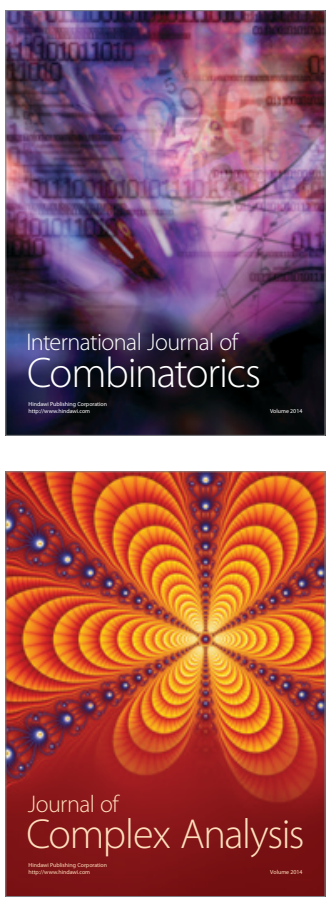

International Journal of

Mathematics and

Mathematical

Sciences
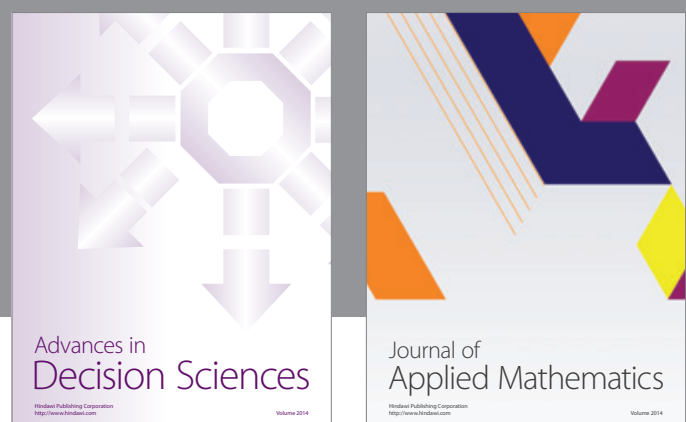

Journal of

Applied Mathematics
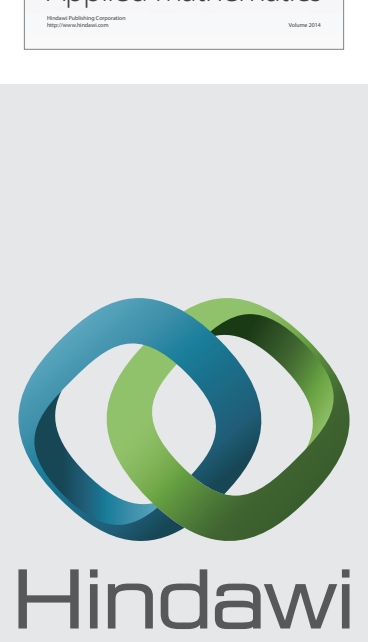

Submit your manuscripts at http://www.hindawi.com
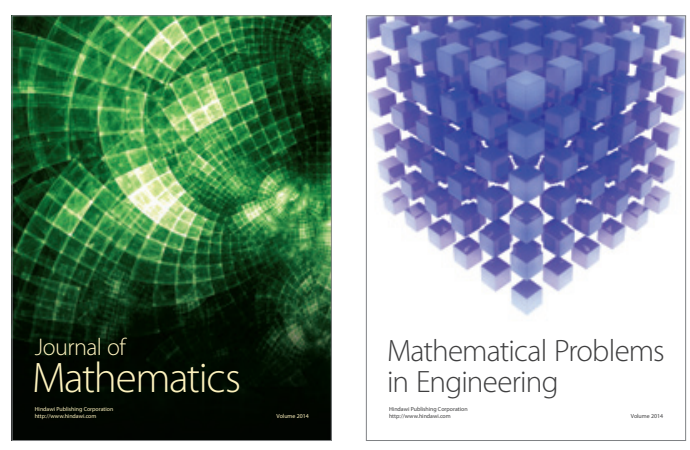

Mathematical Problems in Engineering
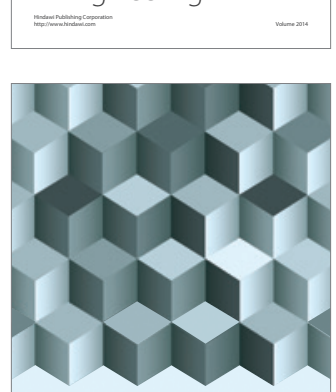

Journal of

Function Spaces
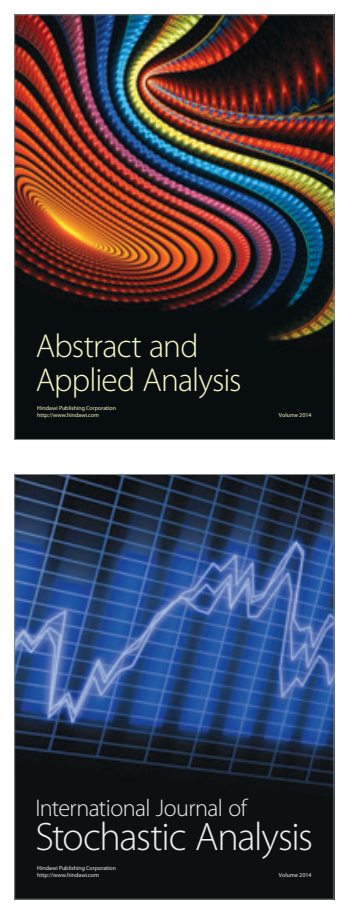

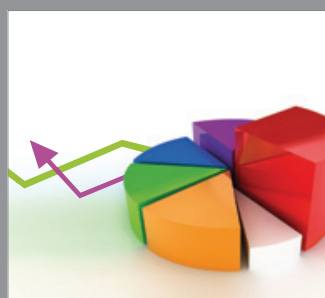

ournal of

Probability and Statistics

Promensencen
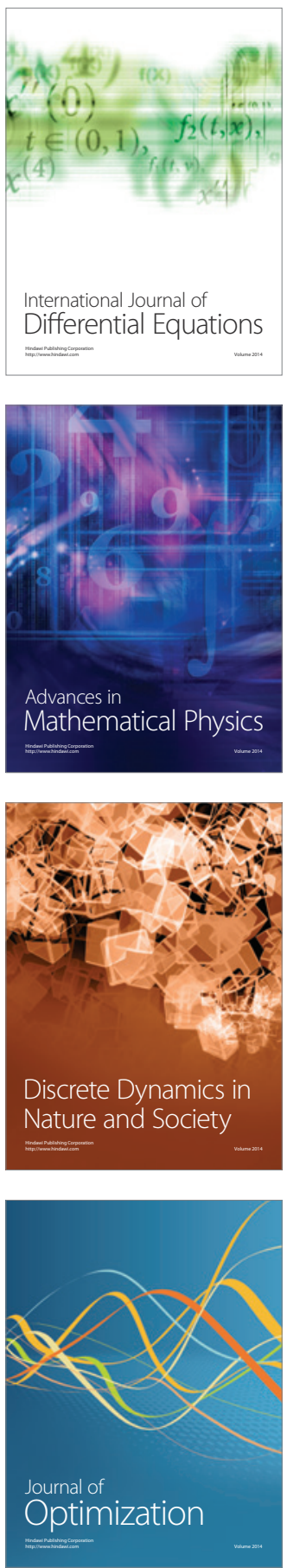